\title{
RELATO DE CASO: SÍNDROME NEUROLÉPTICA MALIGNA
}

\author{
Talissa Nakada Gardin, Beatriz Gadel Medeiros, Raquel Zacharias, Felipe lankelevich Baracat \\ Universidade do Oeste Paulista - UNOESTE, Faculdade de Medicina, Presidente Prudente, SP. e-mail: \\ talissagardin@hotmail.com
}

\section{RESUMO}

Relatar o caso de uma paciente com Transtorno afetivo bipolar (TAB) em acompanhamento psiquiátrico, que foi diagnosticada três vezes com Síndrome Neuroléptica Maligna (SNM) por diferentes antipsicóticos. Paciente feminina de 57 anos, com diagnostico prévio de TAB, deu entrada no hospital com febre, rigidez muscular, tremores, alterações de comportamento, rebaixamento do nível de consciência, aumento de CPK e leucocitose, que não foram esclarecidos por outra condição médica, suspeitando- se então de Síndrome Neuroléptica Maligna. $\mathrm{O}$ uso de antipsicóticos é imprescindível para várias alterações psiquiátricas, como quadros de mania, transtornos de personalidade e doenças demenciais em geral, sendo imprevisível o aparecimento da SNM. Conclui-se que é necessário o conhecimento aprofundado de doença para o diagnóstico e tratamento precoces e, assim, um melhor prognóstico.

Palavras-chave: síndrome maligna neuroléptica, antipsicóticos, olanzapina, eletroconvulsoterapia, transtorno bipolar.

\section{CASE REPORT: NEUROLEPTIC MALIGNANT SYNDROME}

\section{ABSTRACT}

To report the case of a patient with bipolar affective disorder (BPD) in psychiatric care, who was diagnosed three times with Neuroleptic Malignant Syndrome by different antipsychotics (NMS). A 57-year-old female patient with previous diagnosis of BD was admitted to the hospital with fever, muscle stiffness, tremors, behavioral changes, lowering of consciousness level, increased CPK and leukocytosis, which were not clarified by another medical condition, then suspected of Neuroleptic Malignant Syndrome. The use of antipsychotics is essential for several psychiatric disorders, such as mania, personality disorders and dementia in general, and the onset of NMS is unpredictable. It is concluded that a depth knowledge of the disease is necessary for early diagnosis and treatment, which will result a better prognosis.

Keywords: neuroleptic malignant syndrome, antipsychotics, olanzapine, electroconvulsive therapy, bipolar disorder

\section{INTRODUÇÃO}

A Síndrome Neuroléptica Maligna (SNM) refere-se a uma reação adversa severa de idiossincrasia associada ao uso de antipsicóticos, levodopa, antidepressivos e agentes antihistamínicos. É uma síndrome rara e imprevisível, que tem sido associada com os antipsicóticos de primeira geração (típicos) e de segunda geração (atípicos) ${ }^{1}$. Os antipsicóticos são antagonistas de dopamina e estão entre as drogas mais relacionadas à SNM, provavelmente por serem as mais prescritas para neuroleptização rápida e de alta dosagem ${ }^{2}$.

De acordo com o DSM-V (Diagnostic and Statistical Manual of Mental Disorders, 5 Edition) ${ }^{3}$, os critérios utilizados para o diagnóstico de SNM seria a presença da rigidez muscular grave e hipertermia (temperatura de $37^{\circ} \mathrm{C}$, $38^{\circ} \mathrm{OC}$ 
e 40 C) relacionada com a administração recente de neurolépticos, com associação de dois ou mais sinais, sintomas ou achados laboratoriais como: diaforese, disfagia, tremor, incontinência, confusão ou coma, mutismo, taquicardia, taquipneia, pressão arterial elevada ou instável, leucocitose, CPK elevada (acima de 10.000$40.000 \mathrm{U} / \mathrm{L}$ ), ALT e AST elevadas, urina com proteinúria ou mioglobinúria, que não sejam esclarecidos por outra condição médica ou por uso de drogas.

Com a suspeita de SNM, a medicação antipsicótica deverá ser suspensa imediatamente, sendo esta considerada a mais importante e decisiva intervenção médica. Para o tratamento específico, podem ser utilizados os agonistas dopaminérgicos (Bromocriptina ou Amantadina) ou relaxante muscular (Dantrolene) $)^{4}$. A literatura recomenda também a descontinuação do lítio se usado em associação. O paciente deverá ser encaminhado para Unidade de Tratamento Intensivo (UTI) para monitoramento e medidas de suporte clínico ${ }^{5}$. As principais complicações a serem evitadas são os distúrbios respiratórios e a insuficiência renal, associadas com coagulação intravascular disseminada (CIVD), instabilidade hemodinâmica e rabdomiólise, além das sequelas neuropsiquiátricas e físicas de longo prazo ${ }^{6}$.

Os antipsicóticos são amplamente prescritos para diversas patologias psiquiátricas, incluindo quadros depressivos e de mania, transtornos de personalidade, alguns sintomas de demência e para casos específicos de alteração comportamental ${ }^{7}$. Desse modo, o conhecimento por parte da equipe médica sobre a SNM possibilita um rápido reconhecimento dos sinais e sintomas, bem como imediato diagnóstico e intervenção, sendo decisivos para melhor prognóstico desses pacientes ${ }^{8}$.

Nesse contexto, o objetivo desse trabalho é relatar o caso de uma paciente com Transtorno Afetivo Bipolar (TAB), em acompanhamento psiquiátrico, que foi diagnosticada três vezes com SNM, por diferentes antipsicóticos, visando elucidar condutas clínicas para um diagnóstico mais preciso e precoce.

\section{RELATO DE CASO}

A descrição de divulgação do presente caso foi avaliada e aprovada pelo Comitê de Ética em Pesquisa (CEP) da UNOESTE, sob o protocolo CAAE 02848018.1.0000.5515.

Paciente de 57 anos, sexo feminino,
branca, casada, natural de Aruana-PR, procedente de Caiuá-SP, evangélica e dor lar. Deu entrada na unidade de atenção intensiva em saúde mental de um hospital de Presidente Prudente, acompanhada de sua filha com o relato de que há 10 dias paciente está agitada, com excesso de energia, há três noites sem dormir, logorréica, fazendo gastos excessivos e desnecessários. Apresentou alteração da sensopercepção e alucinações visuais. No último dia, apresentou febre e dificuldade para deambular, com rigidez muscular. No exame psíquico encontrava-se vigil, hipotímica, desorientada globalmente com pensamento lentificado e discurso breve. Relata fazer o uso irregular de Carbonato de lítio (1200 mg/dia), Quetiapina (600 mg/dia), Olanzapina (30 mg/dia), Levomepromazina (100 mg/dia). Tem diagnóstico de Transtorno Afetivo Bipolar (TAB) há 20 anos, sem outras patologias. Nega uso de bebidas alcoólicas, drogas e tabagismo.

$\mathrm{Na}$ história psiquiátrica pregressa, paciente apresentou dois episódios semelhantes nos dois anos anteriores, sendo diagnosticada com Síndrome Neuroléptica Maligna. O primeiro episódio foi em novembro de 2015, em uso de Carbonato de lítio $(600 \mathrm{mg} / \mathrm{dia})$, Haloperidol (10 $\mathrm{mg} / \mathrm{dia}$ ), Neosine (25 mg/dia) e Prometazina ( 25 $\mathrm{mg} / \mathrm{dia}$ ), foi internada no hospital Alan Kardec com quadro de mania, onde apresentou febre 39,4 ㅇ C associada a calafrios, rigidez muscular, tremor e mutismo sendo encaminhada para o hospital, onde foi feito o diagnóstico de SNM ficando internada por 40 dias, recebeu alta após melhora clínica e encaminhada para acompanhamento no ambulatório do mesmo serviço. Em maio de 2016, em uso de Quetiapina (600 mg/dia), Carbonato de Lítio (1200 mg/dia) e Lorazepam (2 $\mathrm{mg} / \mathrm{dia})$, apresentou desorientação, discurso desconexo, diminuição da necessidade de sono, inapetência, adinamia, vômitos e febre $37,9^{\circ} \mathrm{C}$, foi internada novamente, onde teve o diagnóstico do segundo episódio de síndrome neuroléptica maligna.

Durante internação hospitalar do terceiro episódio, a paciente manteve rigidez muscular com tremores e alteração do comportamento e febre $(38,5 \circ \mathrm{C})$, evoluindo com rebaixamento de nível de consciência. Pressão arterial: 150/90 $\mathrm{mmHg}$, frequência respiratória: $23 \mathrm{ipm}$, frequência cardíaca: $108 \mathrm{bpm}$, sendo transferida para sala de emergência e colhido exames laboratoriais que mostraram: CPK $34.854 \mathrm{U} / \mathrm{L}$, Hemograma: leucócitos 11.000, segmentados $72,3 \%$, neutrófilos totais $72,3 \%$, eosinófilos $0 \%$, 
basófilos $0,4 \%$, linfócitos $16,6 \%$ e monócitos $10,7 \%$, sódio $156 \mathrm{mmol} / \mathrm{L}$, cálcio iônico 1,39 $\mathrm{mg} / \mathrm{dL}$, magnésio $2,6 \mathrm{mg} / \mathrm{dL}$, lactato 1,1 , creatinina 1,2, uréia 62, AST 427, ALT 150. Urina1: $\mathrm{pH}$ 6,5, proteínas +, corpos cetônicos +, hemoglobina + , glicose negativa, nitrito positivo e leucócitos urinários de $400.000 \mathrm{~mm} 3$, urocultura com presença de Enterobacter cloacae e hemocultura sem desenvolvimento de bactérias.

Foi realizado a suspensão do antipsicótico Olanzapina, devido à suspeita de um novo episódio de Síndrome Neuroléptica e prescrito antibiótico para infecção do trato urinário (ITU) detectada no exame, antitérmicos de horário, sintomáticos, soro de reposição volêmica, correção de eletrólitos e Bromocriptina $2,5 \mathrm{mg}$ $8 / 8$ em SNE. Mesmo após o tratamento da ITU, e até mesmo troca do antibiótico, a paciente não apresentou melhora, mantendo episódios de febre $\left(38,5^{\circ} \mathrm{C}\right)$ que permaneceram por 10 dias, exame psíquico de difícil realização pois paciente encontrava-se em mutismo. Após estabilização clínica, foi transferida para enfermaria da psiquiatria, onde permaneceu até a melhora dos sintomas psíquicos.

\section{DISCUSSÃO}

Os antipsicóticos, também chamados de neurolépticos, são drogas indicadas principalmente no tratamento e seguimento de psicopatologias, agudas e crônicas, que apresentem agitação psicomotora, distúrbios de comportamento, quadros de mania, delírios, alucinações, e inúmeros outros sintomas decorrentes dos transtornos mentais. São divididos em duas classes, os chamados antipsicóticos típicos e atípicos ${ }^{9}$.

Os antipsicóticos típicos foram os primeiros fármacos que se demonstraram efetivos no tratamento da esquizofrenia. Um antipsicótico atípico é definido pelo seu perfil clínico de ações antipsicóticas iguais sobre os sintomas positivos, porém com poucos sintomas extrapiramidais e menos hiperprolactinemia em comparação aos antipsicóticos típicos ${ }^{10}$.

A maioria dos casos de SNM eram relacionados principalmente com os antipsicóticos típicos, porém hoje já se tem inúmeras evidências científicas de que essa síndrome também pode ser ocasionada por drogas atípicas, como a Clozapina, Risperidona, Quetiapina e Olanzapina ${ }^{11}$. As evidências científicas apontam que os antipsicóticos atípicos melhoram a qualidade de vida dos pacientes, no entanto, os antipsicóticos típicos permanecem como escolha no tratamento devido ao menor custo ${ }^{9}$.

A incidência relacionada aos antipsicóticos típicos varia de $0,02 \%$ a 2,46\%, mas não é bem estabelecida para antipsicóticos atípicos. Quando não realizado o diagnóstico precoce, a SNM é fatal entre $10 \%$ a $20 \%$ dos casos $^{4}$.

Mesmo sendo uma reação adversa aos medicamentos neurolépticos, a SNM apresenta inúmeros fatores de risco, que podem desencadear ou provocar reincidência da síndrome, como episódios prévios, desidratação, agitação, ingesta oral pobre, ambientes com temperatura elevada, estresse ocupacional, umidade e uso concomitante de lítio, agentes anticolinérgicos e alguns antidepressivos ${ }^{11}$.

A fisiopatologia da SNM ainda não é bem definida, pesquisas sugerem como hipótese a redução da atividade da dopamina devido ao bloqueio dos receptores dopaminérigicos D2 em regiões do hipotálamo. Isso resultaria em uma dificuldade em dissipar calor e manutenção da temperatura corporal, levando a hipertermia, além disso a rigidez muscular pode ser explicada por uma toxicidade direta dos neurolépticos ao tecido muscular ${ }^{2,11}$.

O tratamento se baseia em uma terapia de suporte. Apenas a retirada da medicação causadora pode ser suficiente para reverter os sintomas. Além disso, deve ser realizado também expansão volêmica, pois a maioria dos pacientes em fase aguda estão desidratados, controles séricos e correção dos eletrólitos. Não há consenso sobre $\mathrm{o}$ uso de medicamentos específicos para tratamento mas existe alguns estudos que apontam drogas que podem ajudar na recuperação do paciente, como agentes colinérgicos (Bromocriptina e Amantadina), bloqueador neuromuscular (Dantrolene) e benzodiazepínicos ${ }^{2}$.

Uma opção de tratamento seria a Eletroconvulsoterapia (ECT) que pode ser utilizada quando as medidas iniciais, como suspensão dos antipsicóticos, tratamento das complicações (hidratação, manejo dos distúrbios hidroeletrolíticos) e uso de medicamentos (bromocriptina e dantrolene) não apresentarem resposta. Consiste na indução de convulsões generalizadas com duração limitada por meio da passagem de corrente elétrica pelo cérebro. Seu mecanismo de ação ainda não é conhecido, mas estudos demonstram ter uma ação similar aos 
efeitos dos antidepressivos na neurotransmissão, como o mecanismo down-regulation de receptores beta-adrenérgicos e aumento da densidade dos receptores $5-\mathrm{HT} 2$. Supõe-se que a baixa utilização desse método se deva principalmente a enganos e preconceitos em relação à ECT. Quando bem indicada, demonstrou ser um tratamento seguro, eficaz e com baixas taxas de mortalidade, aplicável para uma ampla variedade de transtornos psiquiátricos ${ }^{12}$.

Na SNM é difícil prever e definir qual paciente irá apresentar o quadro por se tratar de uma condição idiossincrática e que não se pode associar diretamente com fatores de risco préexistentes. Por isso, é extremamente importante o acompanhamento do paciente em uso de medicações neurolépticas. Mesmo tendo um difícil diagnóstico, quando feito de maneira precoce e precisa, tem efeitos positivos na redução de possíveis complicações e sequelas neuropsiquiátricas ${ }^{2}$.

\section{CONFLITO DE INTERESSE}

Os autores declaram não haver conflito de interesse que poderiam constituir um impedimento para a publicação deste artigo.

\section{REFERÊNCIAS}

1. Sarkar S, Gupta N. Atypical antipsychotics and neuroleptic malignant syndrome: nuances and pragmatics of the association. B J Psych Bulletin. 2016;41(4): 211-216. https://doi.org/10.1192/pb.bp.116.053736

2. Souza PR, Romes APS, Silva MAF, Coelho DM, Galvão Maria de Lourdes S, Souza Nise AC, Picão Ana Paula. Síndrome Neuroléptica Maligna. Rev Bras Clin Med. 2012;10(5):440-5.

3. American Psychiatric Association. Manual diagnóstico e estatístico de transtornos mentais (DSM-V). 5.ed. Porto Alegre: Artmed; 2014.

4. Medeiros FL, Andrade AG, Hiroce VY, Alves TCTF. Síndrome neuroléptica maligna de paciente em uso de olanzapina. J Bras Psiquiatr. 2008;57(2):145-7. DOI: https://doi.org/10.1590/S004720852008000200012.

5. Marchiori PE, Carvalho NB. Síndrome Neuroléptica Maligna. Neurocienc. 2005;13(3):47. DOI https://doi.org/10.34024/rnc.2005.v13.8809.
6. Lemos J, Mós M, Monteiro A, Henriques P. Síndrome maligno dos neurolépticos - um caso raro de evolução prolongada. Acta Med Port. 2010;23(6): 1155-1159.

7. Lima MVB. Assistência odontológica ao paciente psiquiátrico em uso de neuroléptico. [Monografia]. Especialização em Atenção básica em saúde da família, Universidade Federal de Minas Gerais, 2011. Disponível em: https://www.nescon.medicina.ufmg.br/biblioteca imagem/3982.pdf

8. Hanel RA, Sandmann MC, Kranich M, Bittencourt PRM. Síndrome neuroléptica maligna: relato de caso com recorrência associada ao uso de olanzapina. Arq Neuro-Psiquiatr. 1998;56(4):833-7. DOI: https://doi.org/10.1590/S0004282X1998000500022

9. Oliveira IR. Antipsicóticos atípicos: farmacologia e uso clínico. Rev Bras Psiquiatr. 2000;22(Suppl 1):38-40. DOI: https://doi.org/10.1590/S1516$\underline{44462000000500013}$

10. Stahl SM. Psicofarmacologia: Bases neurocientíficas e aplicações práticas. 4.ed. Rio de Janeiro: Guanabara Koogan; 2014.

11. Mendonça SB, Mendonça GS, Quitete $M$, Range CC, Bravin D. Síndrome neuroléptica maligna em paciente em uso de olanzapina relato de caso. Rev Cient FMC. 2016;11(1):26-9

12. Neury JB Pratica psiquiátrica no hospital geral. 3.ed. Interconsulta e Emergência. Porto Alegre: Artmed; 2009.

13. Perizzolo J, Berlim MT, Szobot CM, Lima AFBS, Schestatsky S, Fleck MPA. Aspectos da prática da eletroconvulsoterapia: uma revisão sistemática. Rev Psiquiatr RS. 2003;25(2):327-34. DOI: https://doi.org/10.1590/S0101$\underline{81082003000200009}$

14. Alvarenga PG, Rigonatti SP. Uso de olanzapina e eletroconvulsoterapia em um paciente com esquizofrenia catatônica refratária e antecedentes de síndrome neuroléptica maligna. Rev Psiquiatr RS. 2005;27(3):324-7. DOI: https://doi.org/10.1590/S010181082005000300012 
15. Souza FGM. Tratamento de manutenção no transtorno bipolar. Rev Deb Psiq. 2003;1(5):2433. DOI: http://dx.doi.org/10.1590/S0101$\underline{60832005000700010}$

16. Trollor JN, Xiaohua C, Chitty K, Sachdev PS. Comparison of neuroleptic malignant syndrome induced by first and second generation antipsychotics. B J Psychol. 2012;201(1):52-6. DOI: https://doi.org/10.1192/bjp.bp.111.105189 